\title{
Le traitement des collocations dans les dictionnaires monolingues de collocations du français et de l'anglais
}

\author{
Agnès Tutin \\ Laboratoire de Linguistique et Didactique des Langues Etrangères et Maternelles \\ (LIDILEM), Université Grenoble 3 \\ agnes.tutin@u-grenoble3.fr
}

\section{Introduction}

Dans les discussions linguistiques autour de la notion de collocation, il est usuel de souligner que ce phénomène linguistique est particulièrement épineux à définir. Williams (2003) mentionne ainsi plaisamment :

Les collocations sont en quelque sorte l'Arlésienne de la linguistique : tout le monde en parle, mais elles restent difficilement saisissables. (Williams, $2003: 33$ )

Paradoxalement, si les linguistes peinent à définir ces objets et à les circonscrire de façon précise, depuis quelques années, les lexicographes - peut-être moins frileux - n'hésitent pas à s'en emparer, à les recenser et à les décrire. Bien entendu, les collocations sont aussi traitées dans les dictionnaires de langue générale, et ont parfois même fait l'objet d'un traitement spécifique comme le champ syntagme ${ }^{1} \mathrm{du}$ Trésor de la Langue Française (Hausmann, 1996 ; Tutin, 2005). Le traitement de ces notions a été étudié de façon détaillée pour l'anglais dans les dictionnaires monolingues (par exemple, Moon, 2008 ; Cowie, 1981). Mais, on a vu fleurir récemment plusieurs ouvrages spécialisés dans le traitement des collocations, qui sont passés en peu de temps de projets de recherche artisanaux, œuvres de quelques chercheurs, à des projets lexicographiques d'envergure, comme en témoignent les récents Oxford Collocations Dictionary for Students of English $(2002$; 2008) ou le Dictionnaire des combinaisons de mots : les synonymes en contexte (Le Fur, 2007) de la maison d'édition Le Robert. Ces réalisations, surtout orientées vers l'enseignement des langues étrangères, semblent témoigner d'une certaine maturité de la notion, qui apparaîtrait suffisamment clairement définie pour qu'un traitement linguistique approfondi puisse être réalisé sur un large plan de la langue. Mais cela est-il véritablement le cas ? Quel contour ces ouvrages donnent-ils à la notion de collocation? Quel modèle en proposent-ils ? Peut-on mettre en évidence un noyau stable, en quelque sorte un prototype de la notion de collocation?

Dans cet article, je propose de répondre à ces questions en examinant six dictionnaires de collocations récents de l'anglais et du français, sur les points suivants : la taille de la nomenclature et les traitements syntaxiques et sémantiques proposés. L'objectif est ici de déterminer dans quelle mesure une conception consensuelle de la notion de collocation émerge et de procéder à un examen détaillé des traitements proposés dans ces ouvrages.

\section{Des dictionnaires récents}

Depuis quelques années, au fur et à mesure que l'intérêt pour la phraséologie (et les collocations) s'étendait, sont apparus des ouvrages spécialisés, les dictionnaires de collocations, tout d'abord pour l'anglais, puis plus récemment pour d'autres langues européennes comme le français, l'allemand ou l'espagnol. Seuls les dictionnaires monolingues de collocations sont abordés ici, mais il existe des ouvrages bilingues spécialisés tout à fait intéressants comme le Langenscheidts Kontextwörterbuch Französisch-Deutsch, le « dictionnaire de contextes Langenscheidt Français-Allemand » de Ilgenfritz et al. (1989).

Dans le cadre de la lexicographie contemporaine, ce sont surtout les besoins liés à l'enseignement des langues étrangères qui sont à l'origine de la conception des dictionnaires de collocations. En effet, la 
maîtrise des collocations - et du langage « préfabriqué » dans son ensemble - apparaît essentielle pour la production en langue 2 (Cf. Granger, 1998; Nesselhauf, 2003 ; Siepmann, 2006a). L'existence de ces ouvrages témoigne donc d'une prise de conscience de ces difficultés en didactique du lexique et d'une certaine maturité de la notion de collocation.

Dans les dernières années, ces dictionnaires sont passés de projets individuels, élaborés de façon artisanale dans un contexte universitaire, à de véritables projets éditoriaux impliquant de grandes équipes de rédacteurs, comme en témoignent les récents dictionnaires de collocations parus aux éditions Oxford pour l'anglais (2002 ; réédité en 2009) ou Robert (2007) pour le français.

C'est tout d'abord dans le monde anglophone que ces ouvrages se sont développés depuis une vingtaine d'années ${ }^{2}$, si l'on excepte le peu connu Kenkyusha's Dictionary of English Collocations de 1958 ${ }^{3}$. Parmi ceux-ci, on peut citer :

- The BBI dictionary of English word combinations (désormais BBI) de Morton Benson, Evelyn Benson et Robert Ilson (première édition: 1986, réédité en 1993 puis en 1997), Amsterdam/Philadelphia : John Benjamins Publishing Company). Il contient, outre les collocations « lexicales», des «collocations grammaticales» qui indiquent les prépositions (ou la valence) accompagnant les mots. Nous n'examinerons ici que les collocations lexicales.

- Le Selected English Collocations de Christina Douglas Kozłowska et Halina Dzierżanowska (1982, 1988 (réed), Varsovie : Państowowe Wydanictwo Naukowe) et English Adverbial Collocations de Christina Douglas Kozłowska (1991, Varsovie: Państowowe Wydanictwo Naukowe). Ces deux ouvrages ont été repris et adaptés dans le LTP Dictionary of selected collocations (désormais LTP) de Jimmie Hill et Michael Lewis (2002, Boston : LTP).

- Le Oxford collocations dictionary for students of English (désormais OCDSE) (Crowther et al. 2002, réédité et augmenté en 2009, McIntosh. Oxford : Oxford University Press) est le dernier né et le plus complet de ces dictionnaires, au niveau de la nomenclature et du traitement des collocations.

Il est intéressant d'observer que, dans les parties introductives de ces ouvrages, la dimension « didactique des langues » est extrêmement présente. Le titre du OCDSE indique même explicitement cet usage didactique : « for students of English » et le LTP et le BBI mentionnent spécifiquement ces applications :

[The LTP Dictionnary od Selected Collocations] is a new kind of dictionary to help learners of English to use the words they know more effectively. (LTP : 6) ${ }^{4}$

The BBI is a specialized dictionary designed to help learners of English find collocations quickly and easily (BBI : IX)

Les auteurs, sauf dans le cadre du BBI, n'hésitent pas, même pour un dictionnaire grand public comme le OCDSE, à utiliser le terme technique de « collocation », peut-être parce que ce terme est bien connu dans la communauté lexicographique anglaise où l'école contextualiste est très influente ${ }^{5}$.

Pour le français, les dictionnaires de collocations sont plus récents et comportent une structure moins complexe (moins de structures décrites, description dans l'ensemble moins détaillée), mais la part du français n'étant pas du tout comparable à celle de l'anglais dans l'édition lexicographique, on peut considérer que cette production est loin d'être négligeable. L'intérêt pour les collocations s'est manifesté récemment dans trois ouvrages spécifiques ${ }^{6}$ :

- Le Dictionnaire collocationnel du français général, de Grobelak, L. (1990) (désormais DCFG). Varsovie, Państwowe Wydawnictwo Naukowe, est, à ma connaissance, le premier né des dictionnaires de collocations du français.

- Le Dictionnaire des cooccurrences (désormais DC), Beauchesne, J. (2001). Montréal, Guérin.

- Le Dictionnaire des combinaisons de mots : les synonymes en contexte (désormais DCM) (sous la direction de Dominique Le Fur, 2007, Paris : Dictionnaire le Robert) est comme le OCDSE, un projet d'envergure impliquant une importante équipe de rédacteurs. 
Ne sera pas intégré ici le Dictionnaire combinatoire du français de Zinglé \& Brobeck-Zinglé (2003) ${ }^{7}$, qui mêle à la fois collocations et expressions figées, et qui donc, ne peut véritablement être considéré comme un dictionnaire de collocations. Pour les ouvrages français, la vocation didactique, en particulier pour le français langue étrangère, apparaît moins marquée que pour les ouvrages anglais. Si elle est explicitement mentionnée pour le DCFG - mais son auteur polonais n'est-il pas enseignant de français ? ${ }^{8}$ - cela n'apparaît pas dans la préface du DCM ni dans le DC, qui semblent plutôt destinés aux locuteurs natifs.

Tel n'est pas le cas de deux dictionnaires plus expérimentaux à vocation ouvertement didactique qui font la part belle aux collocations mais ne leur sont pas exclusivement dédiés: Le Dictionnaire d'Apprentissage du Français Langue Etrangère ou Seconde (DAFLES) ${ }^{9}$ de Verlinde \& Binon, (Verlinde S., Selva Th., Binon J., 2003), est un dictionnaire électronique pour apprenants consultable en ligne, et le Lexique Actif du Français (Mel'čuk et Polguère 2007), qui vulgarise les principes du Dictionnaire Explicatif et Combinatoire (Mel'čuk et al. 1995) où les fonctions lexicales sont traitées par des gloses simplifiées ${ }^{10}$.

Sont également exclues de cette étude les bases de données collocationnelles informatiques comme le dictionnaire des cooccurrences du logiciel d'aide à la rédaction Antidote (société Druide) (décrit dans Charest et al., 2007) ou le Sketch Engine de Kilgariff (Kilgarriff et al., 2004). Ces deux ressources, compilées à partir de larges corpus et de techniques de traitement automatique du langage, présentent les collocations en contexte, en corpus, et exploitent des informations de fréquence tout à fait indispensables pour des applications didactiques (Tutin, 2008a). Ces ressources ne peuvent toutefois pas être considérées comme des ressources lexicographiques classiques, mais plutôt comme des outils d'aide à la rédaction, et n'ont pas été intégrées à ce titre dans cette étude.

\title{
3 Principe d'organisation : une mise en œuvre de la dichotomie base- collocatif
}

Les dictionnaires de collocations anglais et français, conformément aux vœux de Hausmann (1989), sont presque exclusivement organisés autour de la distinction binaire entre base et collocatif, qui apparait finalement institutionnalisée dans ces ouvrages. Pour Hausmann en effet,

\begin{abstract}
[...] il ne suffit pas de voir la collocation comme une combinaison sous contrainte, il faut encore comprendre qu'elle est une combinaison orientée. Nous avons tenu compte de cette orientation en appelant l'un des éléments de la collocation base et l'autre collocatif. En effet, dans la collocation célibataire endurci, le signifié de la base (célibataire) est autonome. La base n'a pas besoin du collocatif (endurci) pour être clairement définie. Il en va tout autrement pour le collocatif qui ne réalise pleinement son signifié qu'en combinaison avec une base (célibataire, pécheur, âme, etc.).

Pour un étudiant étranger, cette différence est essentielle dans la mesure où les bases peuvent s'apprendre isolément, alors que l'apprentissage des collocatifs n'est concevable qu'en collocation. [...] cette différence est essentielle pour un dictionnaire d'écriture, parce que celui qui rédige un texte cherche le collocatif à partir d'une base connue et non inversement. (Hausmann 1989 : 1012)
\end{abstract}

Si la question de la binarité suscite parfois quelques discussions chez les linguistes (elle est par exemple remise en question par Bartsch 2004 et Siepmann 2006 ${ }^{11}$ ) force est de constater qu'elle paraît cependant dans l'ensemble suffisamment opérationnelle pour que les lexicographes l'exploitent dans l'organisation de l'information collocationnelle. Dans tous les dictionnaires recensés plus haut (sauf le DCFG), la collocation est exclusivement mentionnée sous l'entrée de la base avec une liste de collocatifs. Par exemple, dans le DC (Figure 1) et le DCFG (Figure 2), la collocation commander l'admiration apparait sous l'entrée de admiration. Le même type de traitement est proposé pour le OCDSE (Figure 3) ou le BBI (Figure 4), par exemple pour la collocation sincere admiration. L'organisation proposée privilégie ainsi l'encodage ou la production, mais comme on le verra plus loin, le traitement linguistique souvent 
rudimentaire rend parfois difficile cette perspective (à moins que les dictionnaires ne soient destinés à des locuteurs natifs ou non natifs de niveau avancé).

\begin{abstract}
ADMIRATION absolue,affectueuse, attendrie, aveugle, béate, énorme, enthousiaste, éperdue, exaspérée, excessive, facile, fanatique, (non) feinte, fervente, générale, grande, grandissante, idolâtre, imbécile, immense, immodéré, infinie, injustifiée, inquiete, juste, muette, mutuelle, profonde, renouvelée, respectueuse, réticente, sans bornes/réserve, sincère, sotte, totale, universelle, vive. Vouer une $\sim(+a d j)$; avoir $l \sim(+a d j$.$) ; avoir, commander, éprouver, exciter, faire, forcer,$ imposer, inspirer, nourvir, professer, provoquer, ressentir, sentir, soulever, susciter, témoigner $l \%$ de $l \%$ son $\sim$; sacrifier à $l^{\prime} ;$ être, rester dans $l^{2} \sim$; considérer, regarder, parler avec $\sim$; déborder, délirer, être digne/ému/médusé /muet/pâmépénifié plein pris/ravi/rempli/saisi/soulevé/transporté,pleurer,ramper, ravir, remplir, saisir, s'émouvoir, se pâmer, se récrier, s'exclamer, (ne pas) tarir, transporter $d$ ' ; devenir, être, tomber en $\sim$. Une $\sim$ se porte sur/vers $q q n / q q c h$.
\end{abstract}

Figure 1 : L'article de admiration dans le DC

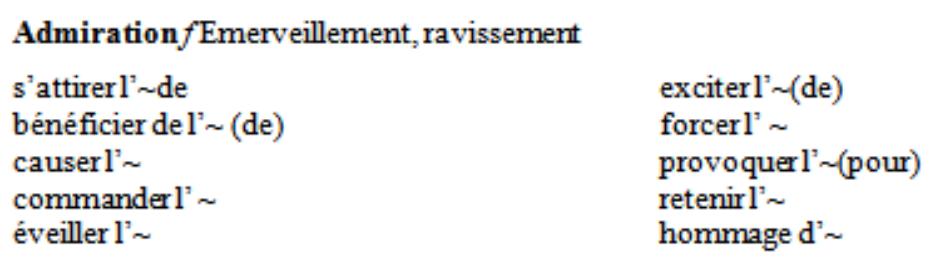

Figure 2 : L'article de admiration dans le DCFG

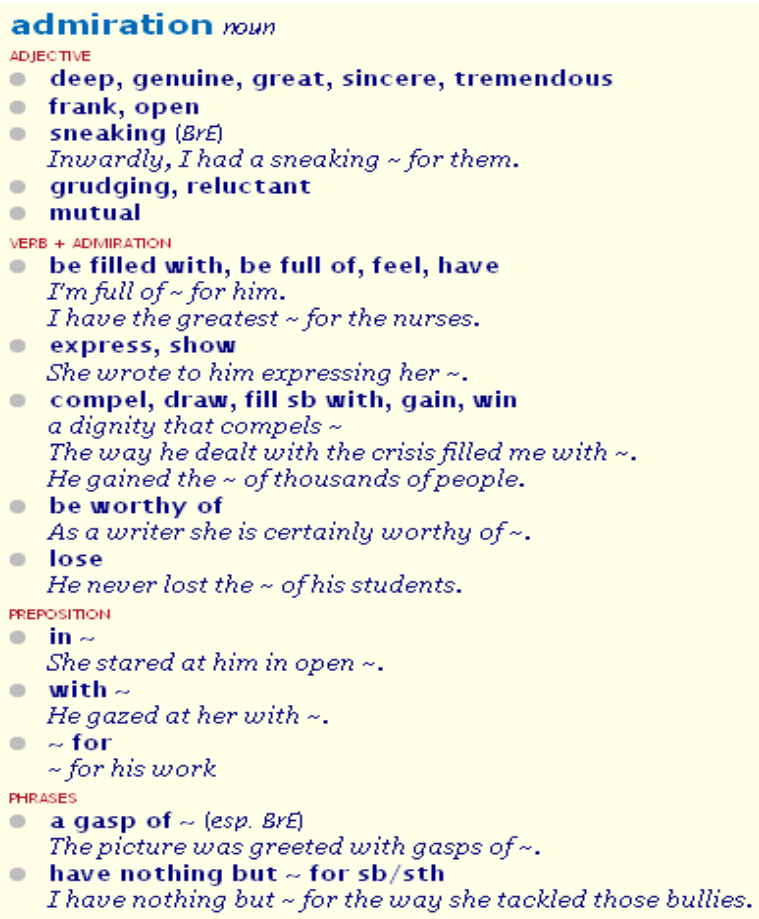

Figure 3 : Article de admiration dans le OCDSE

admiration $n$ 1. to arouse, win; command $\sim 2$. to express; feel, show $\sim 3$. (a) blind; deep, great, sincere, strong, undying; grudging; mutual; secret; ungrudging; universal $\sim 4$. for (he felt great $\sim$ for them) 5 . in, with $\sim$ (to look at smb. with $\sim$ ) 6 . (misc.) (humorous) a mutual $\sim$ society. 
Fig. 4 : Article de admiration dans le BBI

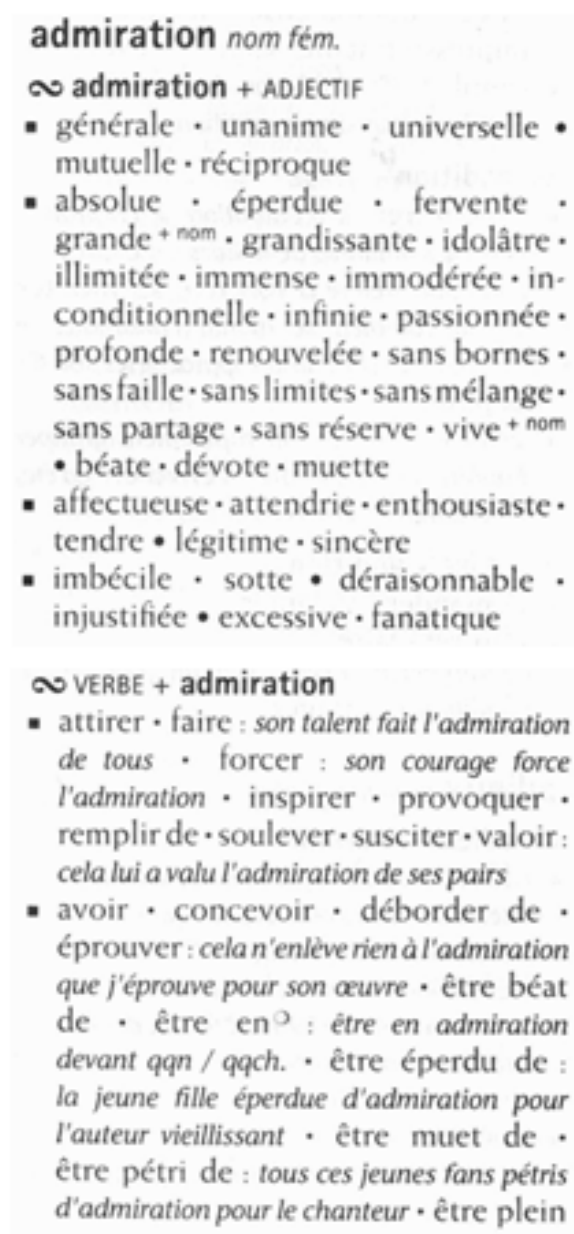

de - être pris de : elle s'était prise d'une vive admiration pour le compositeur - être rempli de · être saisi de · être soulevé de - être transporté de - nourrir : nourrir une admiration pour qqn / qqch. - porter : il lui porte une admiration sans bornes . ressentir * se prendre de • vouer (+ adj.) : elle lui voue une admiration sans limites - conserver - garder : je lui garde toute mon admiration - partager : ils partagent la même admiration pour l'écrivain

- être mêlé de : un effroi méléd'admiration. être teinté de : le sumom qu'ils leur attribuent avec une ironie teintée d'admiration

- avouer - confesser - déclarer - dire exprimer - faire part de - murmurer . proclamer $\cdot$ redire $\cdot$ signifier : elle lui a clairement signifié son admiration

- ne pas cacher : il n'a jamais caché son admiration pour le dictateur / leur audace . ne pas dissimuler - professer : $i$ professe la plus grande admiration pour le cinéaste - témoigner : il lui a témoigné son admiration à maintes reprises

- accroître - renforcer

- être digne de - mériter : une telle maitrise mérite notre admiration

Fig. 5 : Article de admiration dans le DCM

Le traitement à partir du collocatif est choisi par l'ensemble des dictionnaires, sauf le DCFG, qui propose un codage redondant où la collocation est en effet à la fois accessible sous le collocatif et sous la base. Par exemple, la collocation soulever l'enthousiasme est doublement codée dans le dictionnaire : elle apparaît sous l'entrée de soulever dans la partie dédiée aux bases nominales, et dans la partie verbale sous enthousiasme. Cette façon de faire peut être assez pratique pour l'utilisateur, mais elle est peu économique pour un dictionnaire papier. Les versions électroniques des dictionnaires comme celles du OCDSE permettent aussi ce double accès, mais l'accès par le collocatif y est calculé automatiquement, et n'apparaît pas dans la version papier. Par exemple, dans la figure 6, on peut observer sous l'article de pay toutes les bases qui l'ont comme sujet et comme objet. On notera cependant que les différents sens de pay ne sont pas désambiguïsés ici : la liste alphabétique contient aussi bien invoice (facture) que attention ou respect (avec un sens abstrait pour pay). 
Pay is used with these nouns as the subject: BANK, CONSUMER, JOB

Pay is used with these nouns as the object: ADVANCE, ARREARS, ATENTION, BALANCE, BILL, BONUS, BRRBE, CALL, CASH, CHARCE, CHECK, CLAIM, COMMISSION, COMPENSATION, COMPLIMENT, CONTRIEUTION, COST, CREDITOR, DAMACE, DEET, DEPOSIT, DIFFERENCE, DIVIDEND, DOWURY, DUTY EMPLOYEE, EQUIUALENT, EXPENSE, FARE, FEE, FINE, FORTUNE, INSTALMENT, INSURANCE, INTEREST, INVOICE, MAINTENANCE, MERCENARY, MINIMUM, MONEY, MORTCAGE, PENALIT, PENANCE, PENSION, POSTACE, PREMIUM, RANSOM, RATE RECOMPENSE, REFUND, RECARD, RENT, RESPECT, REWUARD, ROYALTY, SALARY, SETLEMENT, STAFE, SUBSCRIPTON, SUBSIDY, SUM, SUPPLEMENT, SUPPORT, SURCHARGE, TARIFE, TAX, TOLL, TOTAL, TRIBUTE, TUITION, WISIT, WWACE, WOORKER

Figure 6 : Accès à la collocation par l'entrée du collocatif : les renvois dans le OCDSE

\section{$4 \quad$ Nomenclature et types de collocations recensées.}

Bien entendu, les dictionnaires mentionnés, de par leur mode de production, artisanal ou « industriel », ont des nomenclatures de tailles variées. Les entreprises lexicographiques récentes comme le OCDSE et le DCM sont les ouvrages les plus volumineux, mais on remarquera que le DCM, s'il comporte un nombre impressionnant de collocations, a une nomenclature - d'ailleurs exclusivement nominale - assez restreinte de 2600 noms. Les auteurs ont privilégié pour cet ouvrage un ensemble important de collocations pour une nomenclature réduite, choix d'ailleurs comparable à celui du OCDSE qui se cantonne aux 9000 mots les plus courants de l'anglais, mais avec une moindre proportion de collocations par mot-base. Dans le BBI au contraire, la nomenclature est vaste (18000 entrées) pour un nombre relativement faible de collocations $\left(90000^{12}\right)$. Le tableau 1 résume ces observations.

\begin{tabular}{|l|c|c|c|}
\hline $\begin{array}{l}\text { BBI dictionary of } \\
\text { English word } \\
\text { combinations (1997) }\end{array}$ & 18000 bases & $\begin{array}{c}90000 \text { collocations (et } \\
\text { colligations) }\end{array}$ & $\begin{array}{c}19 \text { collocations (hors } \\
\text { collocations } \\
\text { grammaticales) }\end{array}$ \\
\hline $\begin{array}{l}\text { LTP Dictionary of } \\
\text { selected collocations } \\
\text { (2002) }\end{array}$ & 3200 bases & 55000 collocations & 31 collocations \\
\hline $\begin{array}{l}\text { Oxford collocations } \\
\text { dictionary for students } \\
\text { of English (2009) }\end{array}$ & 9000 bases & 250000 collocations & 28 collocations \\
\hline $\begin{array}{l}\text { Dictionnaire } \\
\text { collocationnel du } \\
\text { français général (1990) }\end{array}$ & Non mentionné & $\begin{array}{l}30000 \text { collocations, dont } \\
\text { beaucoup apparaissent à } \\
\text { la fois sous la base et } \\
\text { sous le collocatif. }\end{array}$ & 10 collocations \\
\hline $\begin{array}{l}\text { Dictionnaire des } \\
\text { cooccurrences (2001) }\end{array}$ & 4200 bases. & Non mentionné & 91 collocations \\
\hline $\begin{array}{l}\text { Dictionnaire des } \\
\text { combinaisons de mots } \\
\text { (2007) }\end{array}$ & 2600 bases & 160000 collocations & 92 collocations \\
\hline
\end{tabular}

Tableau 1 : Taille des nomenclatures dans les dictionnaires monolingues de collocations du français et de l'anglais

En ce qui concerne les catégories syntaxiques des entrées, on observe une différence nette entre les deux langues: les dictionnaires de collocations du français ne traitent pour l'instant que les bases nominales, qui sont, il est vrai, les plus riches en collocations ${ }^{14}$. Cela est lié au fait que la plupart des collocations 
sont inscrites dans un schéma prédicat-argument, où le prédicat est le collocatif et l'argument la base (Cf. Tutin, 2008b). Les arguments nominaux étant plus fréquents que les arguments verbaux ou adjectivaux, les collocations à base nominale apparaissent donc plus productives. Pour les bases nominales, les dictionnaires français et anglais intègrent pour la plupart des collocatifs verbaux (prêter attention, to command admiration), adjectivaux (colère noire, sincere admiration) ou nominaux sauf pour le DC (bourrasque de vent, gasp of admiration). On notera que les dictionnaires proposent généralement une définition fonctionnelle des catégories de collocatifs : ainsi, on relève par exemple dans le DCM les collocatifs sans bornes, sans faille, sans limites dans la catégorie « admiration + ADJECTIF » alors que les collocatifs être muet de, être pris de, être saisi de sont incluses dans la classe "VERBE + admiration ». Ce type de traitement paraît tout à fit adapté dans une perspective onomasiologique : l'usager se préoccupe peu, lorsqu'il souhaite cibler une expression adéquate, de la catégorie grammaticale de cette dernière ; il s'intéresse plutôt à la fonction qu'elle remplit (qualifiant, par exemple).

Par ailleurs, le BBI et le OCDSE proposent également des prépositions comme collocatifs pour les bases nominales : des prépositions pleines (non régies) par exemple in admiration, with admiration ou des prépositions régies comme admiration for (Cf. Figures 3 et 4). Les auteurs du BBI considèrent toutes ces structures comme des collocations grammaticales. Ce traitement des prépositions dans ces dictionnaires est contestable: le OCDSE traite les prépositions régies comme des collocatifs, et le BBI intègre les prépositions pleines dans les collocations grammaticales. On peut considérer que du point de vue sémantique, seules les prépositions pleines (non régies) peuvent avoir un statut de collocatif à part entière, les prépositions régies devant être intégrées aux informations de combinatoire grammaticale plutôt que lexicale, comme dans les modèles lexicographiques basées sur le Dictionnaire Explicatif et Combinatoire ou le Dictionnaire du Français Usuel (Picoche \& Rolland, 2002).

Contrairement aux dictionnaires français, le BBI, le OCDSE et LTP intègrent également des bases adjectivales et verbales. Par exemple, le verbe admire est traité dans les trois dictionnaires (figures 7, 8 et 9) et l'adjectif angry dans le OCDSE et le BBI (Fig. 10 et 11). Le LTP se restreint aux collocatifs adverbiaux pour les adjectifs et les verbes (en particulier des qualifiants d'intensité, par exemple admire enormously), ainsi que le BBI en ce qui concerne les collocations lexicales. Le OCDSE intègre également des collocatifs verbaux pour les bases adjectivales et nominales (to look angry, to have to admire).

Enfin, le OCDSE inclut un autre type d'expressions dans ses entrées appelé «phrases » qui inclut aussi bien des collocations atypiques excédant deux éléments que des expressions un peu plus figées (par exemple, have nothing but admiration s.v. admiration, I cant'help admiring s.v. admire). La définition un peu vague est simplement : « short phrases including the headword» (OCDSE : VII).

\title{
ADMIRE
}

admire sb enormously, greatly, secretly, tremendously

genuinely, greatly admire

Figure $7:$ L'article de admire dans le LTP

\author{
admire v. 1. to preatly, very much. 2. (D;tr.) to $\sim a$ (we $~$ admire her as a devoted teacher) 3 . (D;tr.) \\ to wor (we wher for her tact) 4 . (K) they all $n$ his behaving in that manner.
}

Figure 8 : L'article de admire dans le BBI 


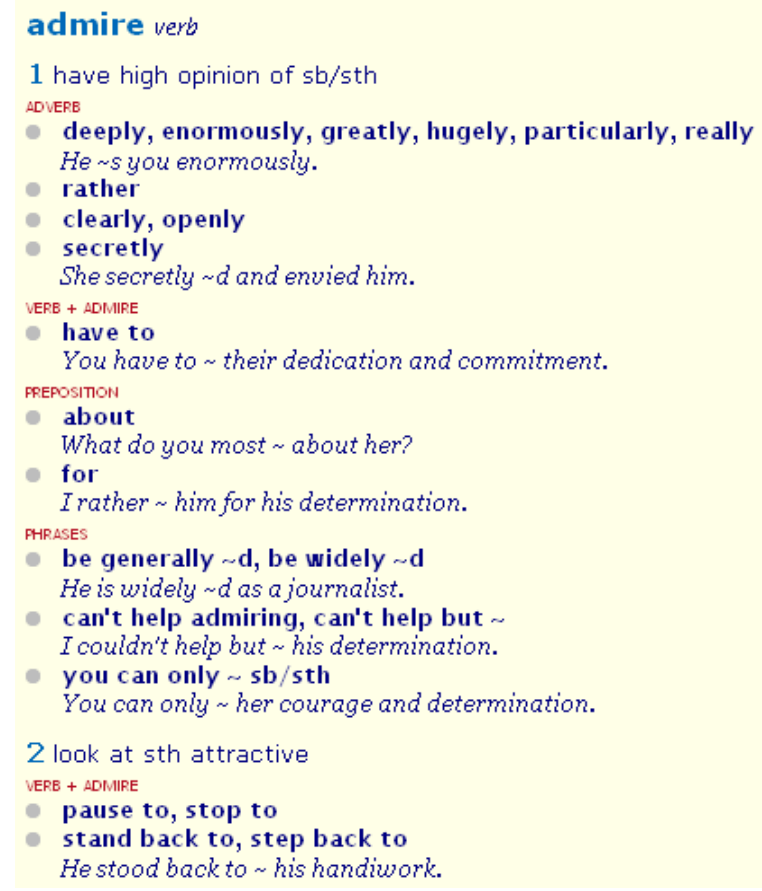

Figure 9 : L'article de admire dans le OCDSE

angry adj. 1 . to become, get $\sim 2$. $\sim$ about, at, over smt.(they were $\sim$ about the changes; we were $\sim$ at being disturbed; they get $\sim$ over every trifle) $3 . \sim$ at (esp. AE), with smb. (he was $\sim$ at his neighbor; she was $\sim$ with me for being late) $4 . \sim$ to $+\inf$. (I was $\sim$ to learn of his refusal to help) 5. $\sim$ that + clause (we wera $\sim$ that our request had been rejected)

Figure 10 : L'article de angry dans le BBI

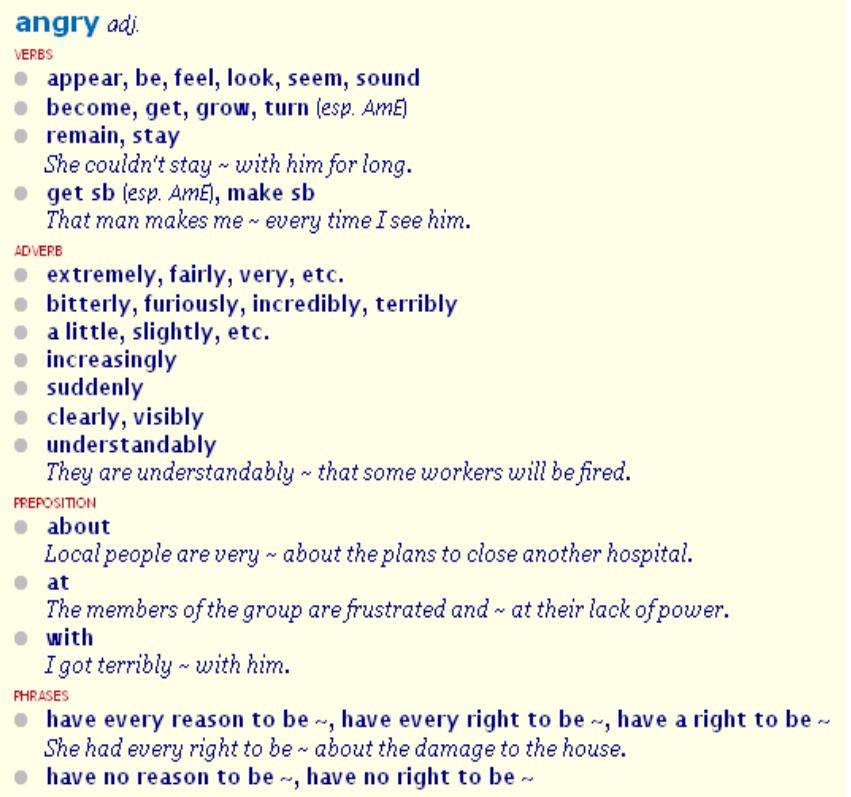

Figure 11 : L'article de admire dans le OCDSE 
Le tableau 2 récapitule ces structures, et donne quelques exemples.

\begin{tabular}{|c|c|c|}
\hline Dictionnaire & Bases et structures traitées & Remarques \\
\hline $\begin{array}{l}\text { BBI dictionary of } \\
\text { English word } \\
\text { combinations (1997) }\end{array}$ & $\begin{array}{l}\text { - Collocations lexicales } \\
\text { - bases nominales } \\
. \mathrm{V}+(\text { Prep }+) \mathrm{N} \text { : commit a suicide } \\
. \text { Adj }+\mathrm{N} \\
. \mathrm{N}+\mathrm{V} \\
. \mathrm{N} \text { Prep }+\mathrm{N}: \text { swarm of bees } \\
\text { - bases verbales } \\
. \text { Adv }+\mathrm{V} \\
\text { - bases adjectivales } \\
\text {. Adv }+\mathrm{A} \\
\text { - Collocations grammaticales : } \\
\text {. contiennent les prépositions et } \\
\text { conjonctions régies par les bases. }\end{array}$ & $\begin{array}{l}\text { - Collocations grammaticales et collocations } \\
\text { lexicales sont mêlées dans le même article. } \\
\text { - Deux sous types sémantiques dans les } \\
\text { collocations de type } \mathrm{V}+\mathrm{N} \text { : } \\
\text { création/activation et éradication/ } \\
\text { «nullification» }\end{array}$ \\
\hline $\begin{array}{l}\text { LTP Dictionary of } \\
\text { selected collocations } \\
(\mathbf{2 0 0 2})\end{array}$ & $\begin{array}{l}\text { - bases nominales : } \\
\quad-\mathrm{Adj}+\mathrm{N} \\
-\mathrm{V}+\mathrm{N} \\
-\mathrm{N}+\mathrm{V} \\
-\mathrm{N}(+ \text { Prep })+\mathrm{N} \text { : plan of action } \\
\text { - bases verbales : } \\
\quad \text { - Adv }+\mathrm{V} \text { : admire enormously } \\
\text { - bases adjectivales : } \\
\quad \text { - Adv }+\mathrm{A} \text { : extremely anxious }\end{array}$ & $\begin{array}{l}\text { - Les bases verbales et les bases adjectivales } \\
\text { sont décrites dans la même section. }\end{array}$ \\
\hline $\begin{array}{l}\text { Oxford collocations } \\
\text { dictionary for } \\
\text { students of English } \\
(\mathbf{2 0 0 9 )}\end{array}$ & $\begin{array}{l}\text { - bases nominales : } \\
\text { - Adj }+\mathrm{N} \\
\text { - quantifieur }+\mathrm{N}: \text { a beam of light } \\
-\mathrm{V}+\mathrm{N} \\
-\mathrm{N}+\mathrm{V} \\
-\mathrm{N}+\mathrm{N} \text { : a light source } \\
\text { - Prep }+\mathrm{N} \text { : by the light of } \\
\text { - } \mathrm{N}+\text { Prep : the light from the windows } \\
\text { - bases verbales : } \\
\text { - Adv }+\mathrm{V}: \text { choose carefully } \\
-\mathrm{V}+\mathrm{V}: \text { be free to choose } \\
\text { - V }+ \text { Prep : choose between } \\
\text { - bases adjectivales : } \\
\text { - V + A : prove positive } \\
\text { - Adv }+\mathrm{A}: \text { perfectly safe } \\
\text { - A + Prep : safe from attack } \\
\end{array}$ & $\begin{array}{l}\text { - Les structures } \mathrm{N}+\text { Prep, } \mathrm{V}+\text { Prep, } \mathrm{A}+ \\
\text { Prep peuvent être interprétées comme des } \\
\text { collocations grammaticales ou des éléments } \\
\text { de sous-catégorisation. Elles ne sont pas } \\
\text { traditionnellement considérées comme des } \\
\text { collocations lexicales. } \\
\text { - les structures plus atypiques son intégrées } \\
\text { dans la catégorie « phrases » : collocations } \\
\text { coordonnées (safe and sound, pick and } \\
\text { choose), }\end{array}$ \\
\hline $\begin{array}{l}\text { Dictionnaire } \\
\text { collocationnel du } \\
\text { français général } \\
(\mathbf{1 9 9 0 )}\end{array}$ & $\begin{array}{l}\text { - bases nominales : } \\
-\mathrm{V}+(\text { Prep }+) \mathrm{N} \\
-\mathrm{N}+\text { Adj } \\
-\mathrm{N}+\text { Prep }+\mathrm{N}\end{array}$ & \\
\hline $\begin{array}{l}\text { Dictionnaire des } \\
\text { cooccurrences } \\
(2001)\end{array}$ & $\begin{array}{l}\text { - bases nominales : } \\
-\mathrm{V}+(\text { Prep }+) \mathrm{N} \\
-\mathrm{N}+\mathrm{V} \\
-\mathrm{N}+\text { Adj }\end{array}$ & \\
\hline $\begin{array}{l}\text { Dictionnaire des } \\
\text { combinaisons de } \\
\text { mots (2007) }\end{array}$ & $\begin{array}{l}\text { - bases nominales } \\
\quad-N+V \\
-V+(\text { Prep }+) N \\
-N+A \\
-N+\text { Prep }+N\end{array}$ & \\
\hline
\end{tabular}

Tableau 2 : Types de collocations traités dans les dictionnaires de collocations 


\section{Microstructure de l'article}

\subsection{Organisation générale}

Les articles de dictionnaires de collocations examinés sont tous organisés autour de deux principes :

a) un premier classement qui regroupe les collocations par constructions syntaxiques.

b) un second classement sémantique ou les collocatifs sont généralement regroupés par synonymie ou quasi-synonymie pour un sous-ensemble de dictionnaires.

Le regroupement syntaxique peut être complètement implicite - c'est le cas du DC, du DCFG, du BBI et du LTP. Par exemple, dans le DC, c'est le passage du romain à l'italique qui signale le changement de structure, comme on peut le voir dans la figure 1. Dans le BBI, les collocations sont regroupées implicitement par structure (et par analogie sémantique). Dans le OCDSE et le DCM, les différents types de structures sont introduits spécifiquement par un indicateur de catégorie, par exemple «VERBE + admiration» dans le DCM. Tous les dictionnaires de collocations analysés ici adoptent ce premier principe de classement.

A l'intérieur du classement syntaxique, un deuxième classement sémantique (synonymique ou quasisynonymique) est opéré par la moitié des dictionnaires. Le DCM, le OCDSE et le BBI regroupent en effet les collocatifs qui entretiennent une affinité sémantique. Par exemple, les collocatifs adjectivaux de admiration qui mettent en jeu une dimension sociale sont regroupés sous la même puce dans le DCM générale, unanime, universelle, mutuelle, réciproque. Le BBI et le DCM affinent encore le classement synonymique. Ainsi, dans l'exemple précédent, mutuelle et réciproque apparaissent sous la même souspuce, alors que générale, unanime et universelle sont regroupés (cf. Figure 5). Le DCM propose un ordre constant dans la présentation des groupements sémantiques, dont la description rappelle ici les fonctions lexicales du Dictionnaire explicatif et combinatoire :

Les groupes de sens sont eux-mêmes ordonnés selon une progression « naturelle», que nous espérons intuitive. Ainsi, pour les états, les actions et les processus, l'article débute par ce qui exprime la cause et le commencement, pour se terminer par la disparition en passant par l'intensification et l'atténuation, ce qui aide et fait obstacle (CDM : VI).

Dans ce dernier dictionnaire qui a pour sous-titre «les synonymes en contexte», le classement synonymique est très affiné. Le DC adopte quant à lui un classement mixte: alphabétique pour les collocatifs adjectivaux, synonymique pour les constructions verbales.

En cas de polysémie des bases, les entrées sont organisées en sous-entrées (OCDSE, DC, BBI), soit dégroupées (DCM, DCFG, LTP).

Le tableau 3 synthétise ces observations. 


\begin{tabular}{|c|l|l|}
\hline & Classement syntaxique & Classement sémantique \\
\hline $\begin{array}{c}\text { BBI dictionary of English word } \\
\text { combinations (1997) }\end{array}$ & implicite & $\begin{array}{l}\text { regroupement quasi- } \\
\text { synonymique à deux niveaux. }\end{array}$ \\
\hline $\begin{array}{c}\text { LTP Dictionary of selected } \\
\text { collocations (2002) }\end{array}$ & implicite & $\begin{array}{l}\text { Inexistant. Regroupement } \\
\text { alphabétique }\end{array}$ \\
\hline $\begin{array}{c}\text { Oxford collocations dictionary for } \\
\text { students of English (2009) }\end{array}$ & explicite avec des étiquettes & $\begin{array}{l}\text { regroupement synonymique à un } \\
\text { niveau. }\end{array}$ \\
\hline $\begin{array}{c}\text { Dictionnaire collocationnel du } \\
\text { français général (1990) }\end{array}$ & implicite & $\begin{array}{l}\text { Inexistant. Regroupement } \\
\text { alphabétique. }\end{array}$ \\
\hline $\begin{array}{c}\text { Dictionnaire des cooccurrences } \\
\text { (2001) }\end{array}$ & implicite & $\begin{array}{l}\text { Regroupement alphabétique pour } \\
\text { les collocatifs adjectivaux. } \\
\text { Regroupement synonymique } \\
\text { pour les collocatifs verbaux }\end{array}$ \\
\hline $\begin{array}{c}\text { Dictionnaire des combinaisons de } \\
\text { mots (2007) }\end{array}$ & explicite avec des étiquettes & \begin{tabular}{l} 
regroupement synonymique \\
\hline
\end{tabular} \\
\hline
\end{tabular}

Tableau 3 : Organisation des articles

\subsection{Traitement linguistique}

Nous avons donc observé que le traitement sémantique explicite est donc quasiment inexistant dans la plupart des dictionnaires, hormis des précisions sur le sens des mots-bases que le DCFG est le seul à pratiquer $^{15}$ (Cf. figure 2 ci-dessus). Le regroupement des collocatifs par quasi-synonymes dans le BBI, l'OCDSE et e DCM permet d'en faciliter le décodage sémantique. Par exemple, dans le DCM peur bleue qui apparaît assez opaque est associée aux autres collocations peur affreuse, peur atroce, épouvantable, horrible ...), ce qui permet assez facilement d'en comprendre le sens. Dans les ouvrages présentés, il n'y a donc ni glose, ni définition de la collocation en tant que telle. Le DCM, en outre, présente une indication intéressante sur l' «indice de figement» pour des expressions comme prudence de sioux, sourire fendu jusqu'aux oreilles ou peur bleue : Indice de figement : Une petite lune attire l'attention des lecteurs sur la forte cohésion
de certaines combinaisons lexicales. » (DCM, Préface, VII).

Cependant, cette indication apparaît également avec être en admiration (Cf. Figure 5) qui peut être considéré comme compositionnel, et facilement décodable. Peut-être s'agit-il donc surtout d'un indice d'imprédictibilité plutôt que de figement.

L'information morpho-syntaxique est généralement rudimentaire. Les informations de valence (prépositions régies par la collocation, par exemple) sont mentionnées dans le BBI, le OCDSE et le DCM, mais de façon non systématique (le traitement le plus systématique semble être sur ce plan celui du BBI, peut-être parce qu'il intègre aussi les collocations grammaticales et prête une plus grande attention à ces aspects grammaticaux). Les alternances syntaxiques (possibilité pour une collocation de type N-V ou V-N d'apparaître au passif ou dans une construction moyenne : l'angoisse ronge $\Leftrightarrow$ être rongé par l'angoisse) sont rarement mentionnées, hormis dans le $\mathrm{DCM}^{16}$.

Enfin, certains dictionnaires omettent des informations essentielles comme le genre des noms (par exemple, dans le DC, l'entrée de ADMIRATION) qui est pourtant un élément particulièrement difficile à maîtriser en langue étrangère. La position de l'adjectif épithète, un point aussi assez délicat en français langue étrangère, n'est indiquée de façon systématique que dans le DCM. 
Quant aux marques d'usage, elles ne sont présentées que dans la moitié des ouvrages de façon systématique (OCDSE, DCM, BBI), ce qui apparaît ennuyeux pour des ouvrages destinés à l'encodage.

Les exemples, pourtant essentiels dans la compréhension et pour le réemploi des collocations, n'apparaissent que dans la moitié des dictionnaires (OCDSE, BBI, DCM), ce qui apparaît regrettable car, les collocations sont un phénomène éminemment contextuel. Ils sont cependant assez nombreux dans le OCDSE et le DCM.

\section{Les corpus et les méthodes d'extraction utilisés}

Tous les auteurs des dictionnaires revendiquent l'utilisation de corpus, de façon plus ou moins précise, essentiellement constitués de presse et de textes littéraires. La taille du corpus exploité n'est cependant explicitement mentionnée que dans le OCDSE (le volume de 2 milliards de mots est d'ailleurs impressionnant) qui en fait même un argument publicitaire, en quatrième de couverture.

Based on the authority of the Oxford English Corpus. A collection of nearly two billion words of text that shows the words that really do go together. (OCDSE : quatrième de couverture)

La seconde version du OCDSE exploite donc un corpus plus conséquent que la première, basée sur les 100 millions de mots du British National Corpus (Lea \& Runcie 2002).

Seuls les deux dictionnaires relevant de grandes maisons d'édition, le OCDSE et le DCM, ont utilisé, à ma connaissance, des méthodes de linguistique de corpus utilisant des outils informatiques. Le recours à ces méthodes est évidemment lié à la taille et aux moyens de l'équipe éditoriale, ainsi qu'à la date (récente) de publication. Les méthodes d'extraction automatique de collocations n'étaient pas encore au point et facilement disponibles dans les années 90 , et encore moins dans les années 80 . Pour la conception du DCM, un corpus de 500 millions de mots, organisé en sous-corpus (presse généraliste et spécialisée des 6 dernières années, textes littéraires contemporains) a été utilisé, exploité par un outil d'extraction des collocations ${ }^{17}$.

\begin{tabular}{|l|l|}
\hline & \multicolumn{1}{|c|}{ Méthode d'extraction et corpus utilisés } \\
\hline $\begin{array}{l}\text { BBI dictionary of English word } \\
\text { combinations (1997) }\end{array}$ & Non mentionnée dans l'ouvrage \\
\hline $\begin{array}{l}\text { LTP Dictionary of selected collocations } \\
\text { (2002) }\end{array}$ & Corpus de presse \\
\hline $\begin{array}{l}\text { Oxford collocations dictionary for } \\
\text { students of English (2009) }\end{array}$ & Basée sur le Oxford English Corpus (2 milliards de mots) \\
\hline $\begin{array}{l}\text { Dictionnaire collocationnel du français } \\
\text { général (1990) }\end{array}$ & Basée sur un corpus de 2 millions de mots - extraction manuelle(?) \\
\hline Dictionnaire des cooccurrences (2001) & Romans du 19ème et 20ème siècle. Presse et magazines. \\
\hline $\begin{array}{l}\text { Dictionnaire des combinaisons de mots } \\
\text { (2007) }\end{array}$ & $\begin{array}{l}\text { Basée sur un corpus de textes journalistiques et littéraires de 500 } \\
\text { millions de mots. Utilisation d'un concordancier extrayant les } \\
\text { collocations. }\end{array}$ \\
\hline
\end{tabular}

Tableau 4 : Principaux dictionnaires de collocations de l'anglais et du français 


\section{Synthèse}

$\mathrm{Au}$ terme de cet examen des dictionnaires contemporains de collocations de l'anglais et du français, plusieurs tendances fortes se dégagent :

a) Les collocations sont traitées comme des expressions binaires, organisées autour de schémas syntaxiques récurrents et la dichotomie entre base et collocatif est institutionnalisée par l'organisation de l'article autour de l'entrée base.

b) Les collocations prototypiques intègrent une base nominale (schémas V-N ou N-V, N-A ou N prep $\mathrm{N})$. Seuls les dictionnaires de collocations de l'anglais (BBI et OCDSE) intègrent des schémas syntaxiques nettement plus variés, avec des bases verbales, adjectivales et de nombreux types de collocatifs. Le statut de la préposition comme collocatif est incertain, et la distinction entre préposition régie et non régie n'est pas établie dans les dictionnaires qui l'intègrent (Le BBI et le OCDSE).

c) Les traitements linguistiques proposés restent dans l'ensemble rudimentaires sur les plans syntaxiques et sémantiques. Les traitements sémantiques s'appuient essentiellement sur des regroupements synonymiques qui peuvent parfois être assez fins comme dans le DCM. Les ouvrages qui ne proposent pas de regroupement sémantique peuvent davantage être considérés comme des aide-mémoire que des aides à l'encodage. Ces traitements simples semblent montrer que les collocations sont perçues comme des expressions transparentes en décodage, ou en tout cas facilement interprétables par analogie avec d'autres expressions. En ce qui concerne l'usage (marques d'usage et contextualisations), seuls le BBI, le OCDSE et le DCM semblent véritablement performants.

En bref, malgré une offre plus diversifiée et des ouvrages récents plus complets au niveau de la nomenclature et des informations linguistiques plus approfondies (en particulier, le OCDSE et le DCM), l'information fournie dans les dictionnaires de collocations reste sommaire, en particulier par comparaison avec certains dictionnaires phraséologiques comme le Oxford Dictionary of Current Idiomatic English (dirigé par Cowie ; Cf. Cowie et al. 1975, 1983 ; et discussion dans Cowie 1998) qui code de façon assez systématique les alternances lexicales et syntaxiques. Sur le plan sémantique, le traitement par classement synonymique n'apparaît pas toujours très poussé, sauf dans le DCM, malgré l'absence de gloses ou d'étiquettes sémantiques. Comme le remarque Cowie (2007) dans son analyse des dictionnaires de phraséologie de l'anglais, le traitement sémantique reste le point faible de ces ouvrages. On notera cependant que les ouvrages plus expérimentaux comme le Lexique Actif du Français (Mel'čuk \& Polguère, 2007) ou le DAFLES (BLF) proposent un classement sémantique plus fin, inspiré des fonctions lexicales de la lexicologie explicative et combinatoire (Mel'čuk et al, 1995), mais qui n'a pas été encore étendu aux dictionnaires de collocations plus grand public (Contraintes commerciales? Difficultés à didactiser ce métalangage ?). L'information liée à l'usage (fréquence, registres de langue) est encore trop peu développée, ce qui apparaît gênant pour des ouvrages qui sont souvent décrits comme destinés à l'encodage. L'utilisation de corpus électroniques offre des perspectives intéressantes cependant, avec l'emploi possible de sous-corpus diversifiés. De ce point de vue, le projet lexicographique en cours développé par Blumenthal en collaboration avec des linguistes de l'ATILF (Blumenthal, 2007) paraît tout à fait prometteur. Se basant sur un corpus diversifié de textes journalistiques et littéraires et à l'aide de techniques statistiques et de techniques de $\mathrm{TAL}^{18}$, ces chercheurs proposent de décrire le profil combinatoire de 2000 noms du français, en indiquant de façon systématique le degré de spécificité de la collocation et sa fréquence (l'approche est délibérément basée sur l'usage). Sur le plan syntaxique également, les dictionnaires pourraient davantage tirer profit de l'exploitation des corpus électroniques, en extrayant semi-automatiquement les propriétés distributionnelles ou les alternances syntaxiques (Cf. par exemple les travaux en TAL de Ritz \& Heid, 2005; Heid \& Weller, 2008; Tutin, 2004), à condition toutefois de disposer de corpus extrêmement diversifiés.

Il n'en demeure pas moins que ces ouvrages montrent que les collocations font désormais bien partie du paysage de la phraséologie, où elles sont clairement dissociées des expressions idiomatiques figées. 
Comme d'autres notions clés de la linguistique parfois épineuses à définir, elles commencent à faire l'objet de descriptions et de modélisations de plus en plus poussées.

\section{Remerciements}

Je remercie tout particulièrement Mme Dominique Le Fur, des éditions Le Robert qui m'a fourni de nombreuses informations concernant la confection du Dictionnaire des combinaisons de mots.

\section{Références bibliographiques}

\section{Dictionnaires}

BBI : Benson, M., Benson, I. \& Ilson, R. (1997). The BBI dictionary of English word combinations. Amsterdam/Philadelphia: John Benjamins.

Cowie, A.P., Mackin, R. \& McCaig, I.R. (1975/1983). Oxford Dictionary of Current Idiomatic English. Oxford : Oxford University Press.

DAFLES (Dictionnaire d'apprentissage du français langue étrangère et seconde), maintenant (Base Lexique du Français) : ilt.kuleuven.be/blf/

DC : Beauchesne, J. (2001). Dictionnaire des cooccurrences. Montréal : Guérin.

DCFG : Grobelak, L. (1990). Dictionnaire collocationnel du français général. Varsovie : Państwowe Wydawnictwo Naukowe.

DCM : Le Fur, D. (sous la dir.) (2007). Dictionnaire des combinaisons de mots. Paris : Le Robert.

DFU : Picoche, J., Rolland, J. Cl. (2002). Dictionnaire du Français Usuel. Bruxelles : De Boeck, Duculot.

LAF : Mel’čuk I. \& Polguère A. (2007). Lexique actif du français. Louvain la Neuve : De Boeck.

OCDSE : Crowther, J., Dignen, S. \& Lea, D. (dir) (2002). Oxford Collocations Dictionary for Students of English. 1ère édition. Oxford: Oxford University Press. McIntosh, C. (dir) (2009). Oxford Collocations Dictionary for Students of English. 2ème édition. Oxford: Oxford University Press. La deuxième édition a été utilisée dans cette étude.

LTP : Hill, J. \& Lewis, M. (1997, réédité en 2002). LTP Dictionary of selected collocations. Boston : LTP.

TLF : Trésor de la langue française. Dictionnaire de la langue du XIXè et du XXè siècle (1789-1960). Paris : Editions du Centre National de la Recherche Scientifique (t. 1-10). Gallimard (t. 11-16). 1971-1994.

\section{Autres références}

Bartsch, S. (2004). Structural and functional properties of collocations in English: A corpus study of lexical and pragmatic constraints on lexical co-occurrence. Tübingen: Narr.

Blumenthal, P. (2007). A usage-based dictionary of French collocations. In Kawaguchi Y., Takagaki T., Tomimori N. \& Tsutuga Y. (eds). Corpus-Based Perspectives in Linguistics. Amsterdam/Philadelphia: John Benjamins.

Charest S., Brunelle E., Fontaine J., Pelletier B. (2007). Élaboration automatique d'un dictionnaire de cooccurrences grand public. Actes de TALN 2007, Toulouse. 282-292.

Cowie, A.P. (1981). The treatment of collocations and idioms in learners' dictionaries. Applied Linguistics 2, 3. 223235.

Cowie, A.P. (1998). Phraseological Dictionaries: Some East-West Comparisons. In Cowie, A.P. (ed). Phraseology: Theories, Analysis and Applications, 209-227. Oxford: Oxford University Press.

Cowie, A. (2007). English phraseography, in H. Burger, D. Dobrovol'skij, P. Kühn, N. Norrick (eds), Phraseologie/Phraseology : Ein internationales Handbuch zeitgenössissicher Forschung/An internatial Handbook of Contemporary Research, 929-939. Berlin/New York: Walter de Gruyter.

Granger, S. (1998). Prefabricated Patterns in Advanced EFL Writing : Collocations and Formulae, In Cowie A.P., Phraseology. Theory, Analysis, and Applications. Oxford : Clarendon Press. 
Hausmann, F. J. (1989). Le dictionnaire de collocations. In Hausmann F.J., Reichmann O., Wiegand H.E. \& Zgusta L. (eds), Wörterbücher : ein internationales Handbuch zur Lexicographie. Dictionaries. Dictionnaires, 10101019. Berlin/New-York: De Gruyter.

Hausmann, F.J. (1996). La syntagmatique dans le TLF informatisé. In D. Piotrowski (ed), Autour de l'informatisation $d u$ TLF, Actes du Colloque International de Nancy (29-31 mai 1995), 51-77. Paris : Didier.

Heid, U. \& Weller, M. (2008). Tools for Collocation Extraction: Preferences for Active vs. Passive. Proceedings of the 6th International Conference on Language Resources and Evaluation. Marrakech, Morocco.

Heid, U., \& Ritz, J. (2005). Extracting collocations and their contents from corpora. F. Kiefer et al. (eds): Papers in Computational Lexicography: Complex 2005. Hungarian Academy of Sciences, Budapest.

Ilgenfritz, P., Stephan-Gabinel, N. \& Schneider, G. (1989). Langenscheidts Kontextwörterbuch Franzözich-Deutsch. Belin/München: Langenscheidt.

Kilgarriff A, Rychly, P., Smrz, P. \& Tugwell, D. (2004). The Sketch Engine Proc. of Euralex. Lorient, 105-116.

Lea D. \& Runcie, M. (2002). Blunt instruments and Fine Distinctions: a Collocations Dictionary for Students of English. In Braasch A. \& C. Povlsen (eds), Proceedings of the Tenth EURALEX International Congress. Copenhagen: Center for Sprogteknologi, 819-829.

Mel'čuk I., Clas A., Polguère A. (1995). Introduction à la lexicologie explicative et combinatoire. Louvain : Duculot.

Moon, R. (2008). Dictionaries and collocations. In S. Granger et F. Meunier (eds). Phraseology : an Interdisciplinary Perspective, 313-336. Amsterdam/Philadelphia : John Benjamins.

Nesselhauf, N. (2003). The Use of Collocations by Advanced Learners of English and Some Implications for Teaching. Applied Linguistics, 24(2), 223-242.

Siepmann, D. (2006a). Collocations et dictionnaires d'apprentissage onomasiologiques bilingues : questions aux théoriciens et pistes pour l'avenir. Langue Française, n ${ }^{\circ}$ 107, 99-118.

Siepmann, D. (2006b). Collocation, colligation ans encoding dictionaries. Part 2: Lexicographical aspects. International Journal of Lexicography 19(1), 1-39.

Tutin, A. (2004). Pour une modélisation dynamique des collocations dans les textes. Actes d'Euralex. Lorient.

Tutin, A. (2005). Le dictionnaire de collocations est-il indispensable ? Revue Française de Linguistique Appliquée. Dictionnaires : nouvelles approches, nouveaux modèles, Vol. X-2, 31-48.

Tutin, A. (2008a). L'apport des corpus annotés pour l'élaboration semi-automatique d'une base de collocations de la langue scientifique générale. In François Maniez, Pascaline Dury, Nathalie Arlin et Claire Rougemont (eds), Corpus et dictionnaires de langues de spécialité, 45-65. Grenoble: Presses Universitaires de Grenoble.

Tutin, A. (2008b). For an extended definition of lexical collocations. In Bernal, E. \& DeCesaris, J. (eds). Proceedings of the XIII EURALEX International Congress, 1453-1460.

Verlinde, S., Selva, Th. \& Binon J. (2003). Les collocations dans les dictionnaires d'apprentissage: repérage, présentation et accès. In Grossmann, F. \& Tutin, A. (eds), Les collocations : analyse et traitement. 105-115. Amsterdam: De Werelt.

Williams, G. (2003). Les collocations et l'école contextualiste britannique. In Grossmann, F. \& Tutin, A. (eds), Les collocations lexicales : analyse et traitement. Amsterdam : De Werelt.

\footnotetext{
${ }^{1}$ Les « groupes binaires » étaient repérés dans le corpus Frantext à l'aide de techniques statistiques très évoluées pour l'époque (Gorcy et al. 1970), mais sans utiliser le terme de collocation.

${ }^{2}$ Nous n'intégrons pas ici parmi les dictionnaires de collocations le Dictionary of English Collocations de Kjellmer (1994) qui n'adopte pas notre définition restreinte de la collocation.

${ }^{3}$ Ouvrage signalé dans Cowie (2007 :934). Ce dictionnaire de l'anglais a été élaboré par des lexicographes japonais s'intéressant à l'anglais langue étrangère. L'organisation suit déjà celle qui est préconisée par Hausmann (1989) : les vedettes y sont des bases nominales et les collocatifs des verbes, des adjectifs et des prépositions. Les collocatifs y sont présentés dans des phrases d'exemples.
} 


\footnotetext{
${ }^{4}$ C'est moi qui souligne ici.
}

${ }^{5}$ John Sinclair, le «pape » des collocations dans la tradition contextualiste, a lui-même été le rédacteur en chef du Collins-Cobuild.

6 On remarquera que, hormis le dictionnaire collocationnel du français général, dont la diffusion est malheureusement restreinte, aucun titre ne contient le terme de « collocation ».

${ }^{7}$ Zinglé H. et M.-L. Brobeck-Zinglé. (2003). Dictionnaire combinatoire du français. Expressions, locutions et constructions. Paris, La maison du dictionnaire.

${ }^{8}$ Le projet de dictionnaire de collocations de Grobelak semble étroitement lié à des besoins d'enseignement et de traduction. L'auteur constate ainsi que le dictionnaire bilingue ne permet pas nécessairement de choisir le bon terme dans la langue cible. En outre, la principale difficulté est de trouver pour l'apprenant ou le traducteur le « terme qui entre en collocation avec un autre terme » $(\mathrm{p}$ 8). L'auteur a souhaité « fournir un instrument de travail qui servirait à tous les intéressés : professeurs de lycée et d'universités, traducteurs, élèves avancés et étudiants, rédacteurs de publications francophones » (ibid).

${ }^{9}$ Maintenant intégré dans la Base Lexicale du Français (ilt.kuleuven.be/blf/)

${ }^{10}$ Par exemple, le DAFLES utilise les « fonctions lexicales simplifiées » suivantes (Verlinde, Binon \& Selva 2006 : 91), par exemple : Intensification (un gros problème), Multitude (un troupeau de chèvres), verbe support plein début (entamer des négociations).

${ }^{11}$ Ces auteurs considèrent que des expressions comme avoir un geste déplacé ou freshly baked bread sont des collocations tripartites. Ces analyses ne résistent pas à un examen détaillé. Dans le premier cas, il s'agit d'une superposition de collocations (avoir DET geste Modif + geste déplacé), dans le second cas, d'une collocation récursive où freshly baked est aussi un collocatif.

${ }^{12} \mathrm{Ce}$ nombre inclut en outre les collocations grammaticales.

${ }^{13}$ Plusieurs des informations apportées ici m'ont été fournies par Dominique Le Fur, responsable éditoriale du DCM. Je l'en remercie vivement.

${ }^{14}$ Dominique Le Fur, responsable éditoriale du DCM, indique ainsi (communication personnelle) que « - le nom est généralement le mot porteur du concept, de l'idée que veut exprimer le locuteur, - la « détresse lexicale » décrite par M. Haussmann concerne le plus souvent les collocatifs du nom, hors cas de terminologie ou langage spécialisé (ex cliquer sur la souris). »

${ }^{15}$ D'ailleurs de façon assez imprécise parfois. La glose donnée pour admiration ne semble pas véritablement adaptée.

${ }^{16}$ Par exemple, sous l'entrée de angoisse. « ronger (souvent passif) : rongé d'angoisse/par l'angoisse. » (DCM : 44).

${ }^{17}$ Communication personnelle de Mme Dominique Le Fur.

${ }^{18}$ Les chercheurs utilisent un corpus de presse et Frantext de 68 millions de mots, étiqueté à l'aide Treetagger et repèrent les collocations à l'aide de la mesure de log-likelihood. 